\title{
APSIC: Training and fitting amputees during situations of daily living
}

\author{
H. Pillet ${ }^{\mathrm{a}, *}$, X. Drevelle ${ }^{\mathrm{b}}$, X. Bonnet ${ }^{\mathrm{c}}$, C. Villa ${ }^{\mathrm{a}}$, N. Martinet ${ }^{\mathrm{d}}$, C. Sauret ${ }^{\mathrm{b}}$, J. Bascou ${ }^{\mathrm{b}}$, I. Loiret $^{\mathrm{d}}$,

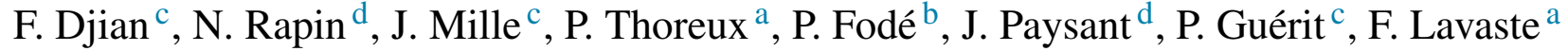 \\ a Arts et Metiers ParisTech, laboratoire de BioMécanique (LBM), 151, boulevard de l'Hôpital, 75013 Paris, France \\ ${ }^{\mathrm{b}}$ CERAH, Institution Nationale des Invalides, 1, route Rombas-Bellevue, 57140 Woippy, France \\ ${ }^{\mathrm{c}}$ Proteor, département recherche et développement, 6, rue de la Redoute, 21250 Seurre, France \\ ${ }^{\mathrm{d}}$ Centre de médecine physique et de réadaptation Louis-Pierquin, IRR-UGECAM Nord-Est, 75, boulevard Lobau, CS 34209 , 54042 Nancy cedex, France
}

\begin{abstract}
Today, the prevalence of major amputation in France can be estimated between 90,000 and 100,000 and the incidence is about 8300 new amputations per year (according to French National Authority for Health estimation). This prevalence is expected to increase in the next decade due to the ageing of the population. Even if prosthetic fitting allows amputee people recovering the walking ability, their autonomy remains limited when crossing obstacles such as slopes, stairs or cross-slopes frequently encountered during outdoors displacements. The aim of the project APSIC was to complete scientific knowledge about adaptation strategies to situations of daily living compared to level walking through an extensive motion analysis study of transtibial and transfemoral amputee compared to non-amputee people. APSIC succeeded in identifying physiologic joint functions and current prosthetic joint limitations in the studied situations, which notably resulted in the design of a prototype of ankle-knee prosthesis adapted to multimodal locomotion of transfemoral amputee. Perspectives of the clinical use of motion analysis within the rehabilitation process were explored and proved to be relevant for personalized approach of motor learning.
\end{abstract}

\section{Introduction}

The amputation of a lower limb, by inducing the loss of several musculoskeletal functions, leads to long-term disability, which alters the quality of life [1]. The restoration of impaired functions by an artificial limb and the rehabilitation of walking ability are essential steps toward reinsertion of the patient in familial, social and work environments. The field of prosthetic components design is very active and prosthetic knees and feet are proposed with a growing level of technology [2]. Conversely, the patients continue to report some difficulties for ambulation, emphasized during outdoor displacements [1]. Indeed, until now, the technological innovations of prosthetic assistive devices were reserved to young and active persons with lower limb loss due to traumatic causes. Therefore, to allow a more massive use of high tech prostheses by the overall population of amputee,

\footnotetext{
* Corresponding author.

E-mail address: helene.pillet@ensam.eu (H. Pillet).
}

efforts must be made in improving both functional capacities of prosthetic components and the specific rehabilitation of people with amputation.

Among the situations of daily living, slopes and stairs had been shown to result in locomotion difficulties [2]. Deep understanding of the locomotion in these situations is accessible from the literature on normal locomotion [3-5]. As a perturbation in the frontal plane, cross-slope walking, even though less investigated in the literature [6], ought to result in complex tridimensional adaptations of the joints. As concerns patients with amputation, the synthesis of literature data from motion analysis studies is much more difficult as the functional level of these patients can be very different depending on multiple factors such as etiology of amputation, muscular capacities, prosthetic fitting.

In this framework, the aim of the project was to develop:

- innovative functions for intelligent prostheses, adapted to multi-modal locomotion; 
- specific rehabilitation for their optimal use.

This contribution relied on biomechanical analysis performed in three situations of daily living: slope ascent and descent, stair ascent and descent and cross-slope walking compared to level walking by using quantitative gait analysis. The scientific outcomes were both:

- to contribute to the understanding of joints functions of nondisabled subjects;

- to identify impaired joint functions and their compensations in transtibial and transfemoral amputee populations.

The article presents:

- the original protocol designed in the project to analyze the locomotion in different situations of the real life;

- an example of biomechanical analysis during the locomotion in stairs and slopes compared to level walking;

- the design process of the ankle-knee prototype dedicated to transfemoral amputee developed in the scope of the project.

\section{Protocol and populations}

\subsection{Material and reproduction of situations of daily living}

Data collection was conducted in two different motion analysis laboratories (INI $\backslash$ CERAH-Créteil, IRR Nancy) with the same instrumentation. Motion capture was made with an optoelectronic system Vicon at $100 \mathrm{~Hz}$ and synchronized with the acquisition of ground reaction forces and moments from two AMTI force plates. Four situations of daily living were simulated using instrumented devices (Fig. 1): two ramps inclined at $5 \%\left(3^{\circ}\right)$ and $12 \%\left(7^{\circ}\right)$, a 4-step staircase (step dimensions riser: $17.5 \mathrm{~cm}$, tread: $28 \mathrm{~cm}$ ) and a cross-slope inclined at $10 \%\left(6^{\circ}\right)$. For the ramps and the cross-slopes devices, modular structures with independent blocks adapted to the geometry of the force plates were designed according to the principles proposed by Dixon and Pearsall [6]. For the stair, the same design as proposed by Della Croce et al. [7] was used.

\subsection{Patients}

Twenty-two transtibial (one female) and 21 transfemoral (two females) amputees participated in the study. Inclusion criteria included independence as a community ambulator without any assistive device. Each patient wore his usual prosthetic device and shoes. A control group consisted of 30 non-amputated subjects who did not have any orthopedic or neurologic disorders. Table 1 summarized general characteristics of the three groups showing the matching between groups.

\subsection{Protocol and walking conditions}

The protocol was approved by the local ethics committee and all participants gave their consent. Patients with amputation benefited from a standard clinical exam prior to the experiments. The whole body was equipped with 54 retroreflective markers placed on specific anatomical landmarks and technical plates. Data collection began by a static acquisition in a freestanding position during which two photographs of the entire body in the sagittal and in the coronal planes were taken. Then, participants were asked to successively perform the following tasks: level walking, ascending and descending ramps (5 and 12\% incline), uphill and downhill cross-slope walking and ascending and descending stairs. For each situation, subjects adopted a self-selected comfortable walking speed. For non-amputated subjects only, a second slower walking speed was asked and was also chosen by the subject. For each of the nine conditions, four successful trials were recorded. In addition, functional evaluation of all amputee subjects was made through PPA-LCI (prosthetic profile amputee-locomotor capacities index) and SF-36 (short form) self-reported questionnaires and a six-minute walk test [8].

\subsection{Modeling and calculation of kinematics and dynamics}

Eighteen body segments could be defined from the marker set. An anatomical reference frame was embedded to each segment according to the definitions proposed by Dumas et al. [9] and Wu et al. [10]. For prosthetic segments, anatomical landmarks and frames were defined by symmetry with the contralateral limb. An anthropometric model of each subject was also built and personalized using the method described by Pillet et al. [11] from the data of the static acquisition and the photographs. This model allows the calculation of body segment inertial parameters useful for inverse dynamic calculation. From the absolute and relative positions of anatomical systems of reference, segment and joint angular positions were assessed. In particular, joint angular positions were obtained from the relative position of the proximal segment of the joint relatively to the distal segment of the joint. The calculation method followed the recommendations of ISB [12], giving: the joint angular position in the transverse plane as the angle of rotation around the $\mathrm{Y}$ axis (distal-proximal) of the distal segment; the joint angular position in the frontal plane as the angle of rotation around the $\mathrm{X}$ axis (floating axis postero-anterior) and the joint angular position in the sagittal plane as the angle of rotation around the $\mathrm{Z}$ axis (medio-lateral) of the proximal segment.

Dynamics of the body during locomotion were computed using the method described by Pillet et al. [11]. They were used in an inverse dynamic procedure to calculate joint forces and moments as the mechanical actions of the proximal segment on the distal segment of the joint. For physiologic joint, the joint moment can be interpreted as the resulting muscular moment in the joint considering that muscles actions are predominant in front of ligaments and contact actions. For prosthetic joint, the joint moment represents the necessary mechanical action exerted by the proximal part on the distal part to ensure the dynamic equilibrium of the distal segment. The joint forces and moments could be projected on the axis of the anatomical frame of the distal segment and interpreted as sagittal force and moment for the $\mathrm{Z}$ axis, frontal force and moment for the $\mathrm{X}$ axis and transverse force and moment for the $\mathrm{Y}$ axis. 


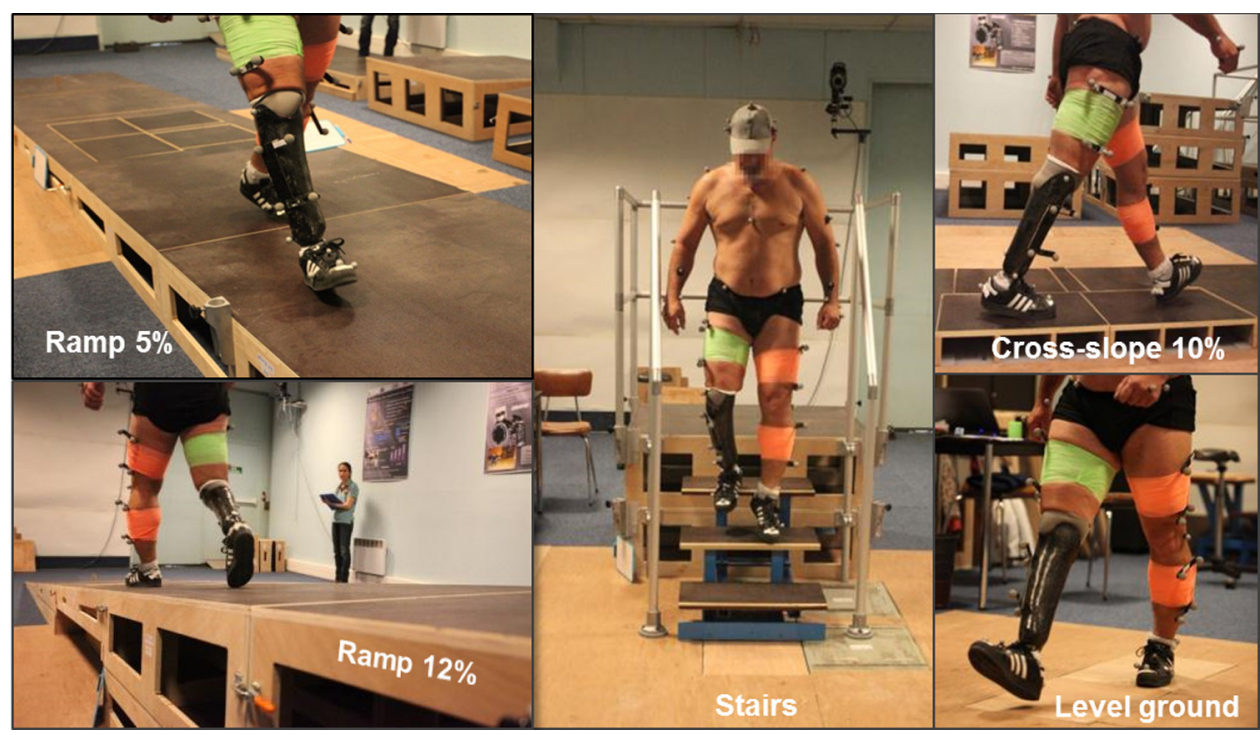

Fig. 1. Four reproduced situations in the gait laboratory.

Joint powers were calculated as the dot product of joint moment and relative angular velocity of the distal segment relatively to the proximal segment of the joint [13]. The joint power could be decomposed in three terms corresponding to the dot product of each component of the vectors joint moment and angular velocity. Each of these terms could be interpreted as sagittal, frontal and transverse joint powers.

\section{Biomechanical analysis: ankle stiffness during slope and stair locomotion}

Ankle stiffness is an example of relevant parameter to characterize ankle function. A part of the difficulties encountered by amputee patients can be attributed to the limited ability of the prosthetic ankle to reproduce the physiologic rollover during the stance phase of gait [14]. In particular, the dorsiflexion movement of the ankle in the single support period of the stance is critical to achieve correct progression in the sagittal plane of the body in the different situations. The quantification of the sagittal stiffness of the physiologic ankle during level walking was shown to be useful to define realistic targets for prosthesis design [15] but has not yet been performed during slopes and stairs locomotion. The evolution of the dorsiflexion of the ankle (Fig. 2a) and the evolution of the ankle moment of dorsiflexion (Fig. 2b) were represented according to the percentage of gait cycle for each condition. To extract the ankle rollover during the stance phase, the part lying from the maximal plantarflexion of the ankle to the maximal dorsiflexion of the ankle was isolated and ankle moment was represented as a function of ankle angle during this subpart of the stance (Fig. 2c). For each subject and each condition, the ankle stiffness could be evaluated at each instant of time as the coefficient linking the ankle angle to the ankle moment. For non-amputee subjects, as illustrated on Fig. 2c, ankle moment $v s$. ankle angle curves could be divided in two parts during which ankle stiffness remained nearly constant. This separation led to compute two values of ankle stiffness K1 and $\mathrm{K} 2$. In addition, a global coefficient $\mathrm{K}$ was assessed as the average of the values of the ankle stiffness all along the ankle rollover. Table 2 gives the averaged values of $\mathrm{K} 1, \mathrm{~K} 2$ and $\mathrm{K}$ for the control group.

The results show that the ankle stiffness of non-amputee people evolved in the different walking conditions (Fig. 3). It globally decreased when descending slopes and stairs. The bilinear behavior of the ankle intensified when climbing slopes compared to level walking with a decreased stiffness at the beginning of the dorsiflexion motion and an increased stiffness at the end (Fig. 3).

To understand the limitations due to the amputation, the ankle stiffness was calculated using the same method for the population of transfemoral amputee. Due to the pattern of the ankle moment/angle evolution in this population, only the global stiffness $\mathrm{K}$ was assessed. The stiffness of the prosthetic ankle was

Table 1

General characteristics repartition (age, height, weight) and averaged walking velocity on level ground for the three studied populations.

\begin{tabular}{|c|c|c|c|c|c|c|c|c|c|c|c|c|}
\hline & \multicolumn{4}{|c|}{$\begin{array}{l}\text { Control group } \\
n=30\end{array}$} & \multicolumn{4}{|c|}{$\begin{array}{l}\text { Transfemoral amputee } \\
n=21\end{array}$} & \multicolumn{4}{|c|}{$\begin{array}{l}\text { Transtibial amputee } \\
n=22\end{array}$} \\
\hline & Moy & Std & Min & $\operatorname{Max}$ & Moy & Std & Min & $\operatorname{Max}$ & Moy & Std & Min & $\operatorname{Max}$ \\
\hline Height $(\mathrm{cm})$ & 173 & 10 & 155 & 192 & 175 & 8 & 161 & 191 & 176 & 7 & 162 & 193 \\
\hline Weight (kg) & 69 & 13 & 46 & 111 & 76 & 13 & 50 & 111 & 85 & 16 & 54 & 110 \\
\hline Walking speed $(\mathrm{m} / \mathrm{s})$ & 1.33 & 0.13 & 1.02 & 1.69 & 1.22 & 0.14 & 0.97 & 1.47 & 1.2 & 0.19 & 0.77 & 1.48 \\
\hline
\end{tabular}



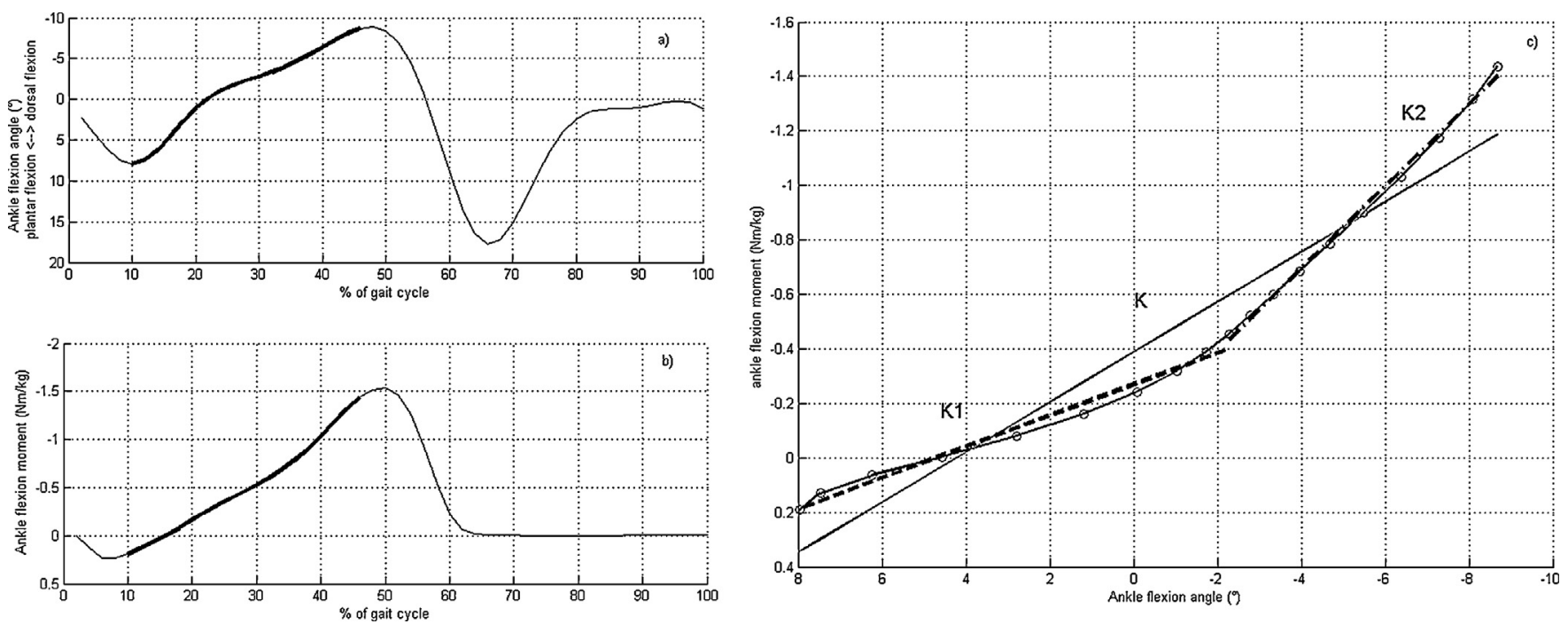

Fig. 2. Quantification of the ankle stiffness for one non-amputee subject: a: pattern of evolution of the ankle angle according to the percentage of gait cycle; b: pattern of evolution of the ankle moment according to the percentage of gait cycle; c: evolution of the ankle moment as a function of the ankle angle, representation of $\mathrm{K}$ the averaged ankle stiffness during the dorsiflexion movement of the ankle, $\mathrm{K} 1$ the averaged ankle stiffness during the first part of the dorsiflexion movement and $\mathrm{K} 2$ the averaged ankle stiffness during the second part of the dorsiflexion movement.

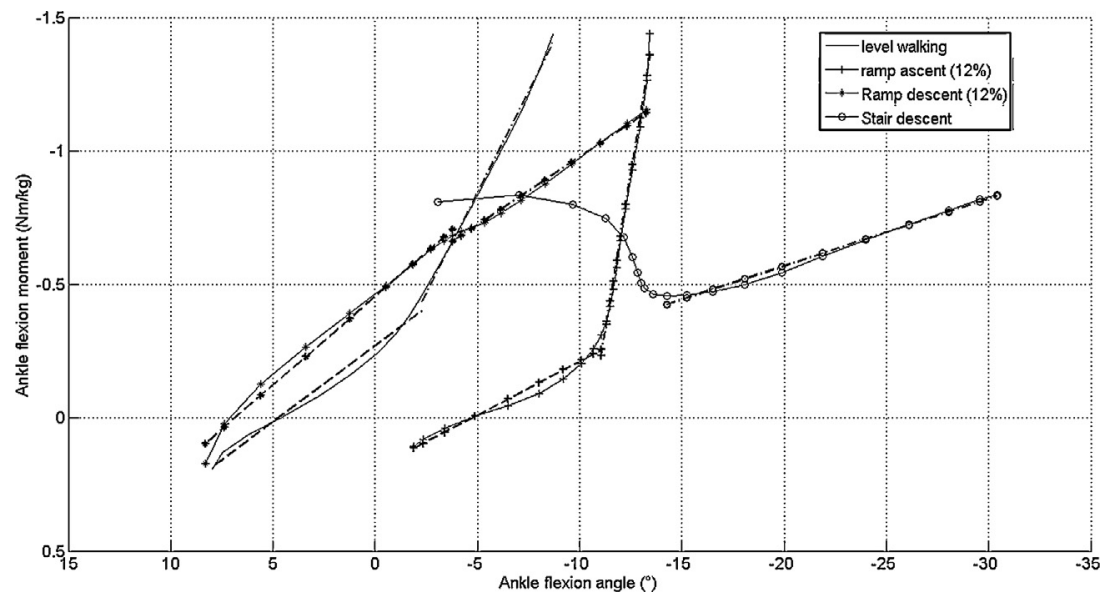

Fig. 3. Average patterns of evolution of the ankle moment as a function of ankle angle for the population of non-amputee subjects for all walking conditions - lines represents the linear approximations of both parts of each curve which slopes are the estimated ankle stiffnesses over the period.

shown to be constant characterizing the mechanical behavior of the component and independent on the crossed situations (average value: $0.08 \mathrm{Nm} / \mathrm{kg} /{ }^{\circ} ; \mathrm{SD}: 0.02 \mathrm{Nm} / \mathrm{kg} /{ }^{\circ}$ ).

Table 2

Values (average and standard deviation) of $\mathrm{K}$ averaged ankle stiffness during the whole dorsiflexion movement, $\mathrm{K} 1$ averaged ankle stiffness during the first part of the dorsiflexion movement, $\mathrm{K} 2$ averaged ankle stiffness during the first part of the dorsiflexion movement for the population of non-amputee subjects.

\begin{tabular}{lllllll}
\hline Mean; SD & $\mathrm{K}$ & $\left(\mathrm{Nm} / \mathrm{kg} /{ }^{\circ}\right)$ & $\mathrm{K} 1$ & $\left(\mathrm{Nm} / \mathrm{kg} /{ }^{\circ}\right)$ & $\mathrm{K} 2$ & $\left(\mathrm{Nm} / \mathrm{kg} /^{\circ}\right)$ \\
\hline Level walking & 0.09 & 0.03 & 0.06 & 0.02 & 0.15 & 0.05 \\
Ramp descent (5\%) & 0.08 & 0.02 & 0.07 & 0.02 & 0.11 & 0.09 \\
Ramp descent (12\%) & 0.06 & 0.03 & 0.07 & 0.02 & 0.06 & 0.07 \\
Ramp ascent (5\%) & 0.09 & 0.02 & 0.04 & 0.02 & 0.20 & 0.10 \\
Ramp ascent (12\%) & 0.09 & 0.02 & 0.04 & 0.02 & 0.31 & 0.19 \\
Stair descent & NA & NA & NA & NA & 0.03 & 0.03 \\
\hline
\end{tabular}

NA: not available.

\section{Prosthetic design: prototype of ankle-knee system}

This part seeks to illustrate how gait analysis data could be used in the design process of a prototype of ankle-knee prosthesis for transfemoral amputee.

Prosthetic design is driven by the aim of mimicking physiological joints behavior. From this point of view, artificial elements are used to reproduce some specific feature of the natural joint. As an example, equivalent ankle stiffness can be obtained by using leaf spring. The adaptation of the mechanical prosthetic elements between different situations is a major issue and necessitates the definition of reliable targets from the analysis of normal gait in real life situations as for example the evolution of the ankle stiffness in these situations. The understanding of the actual limits of current prosthetic components and of the compensation mechanisms used by amputee people is also essential. For example, the constant stiffness of prosthetic 

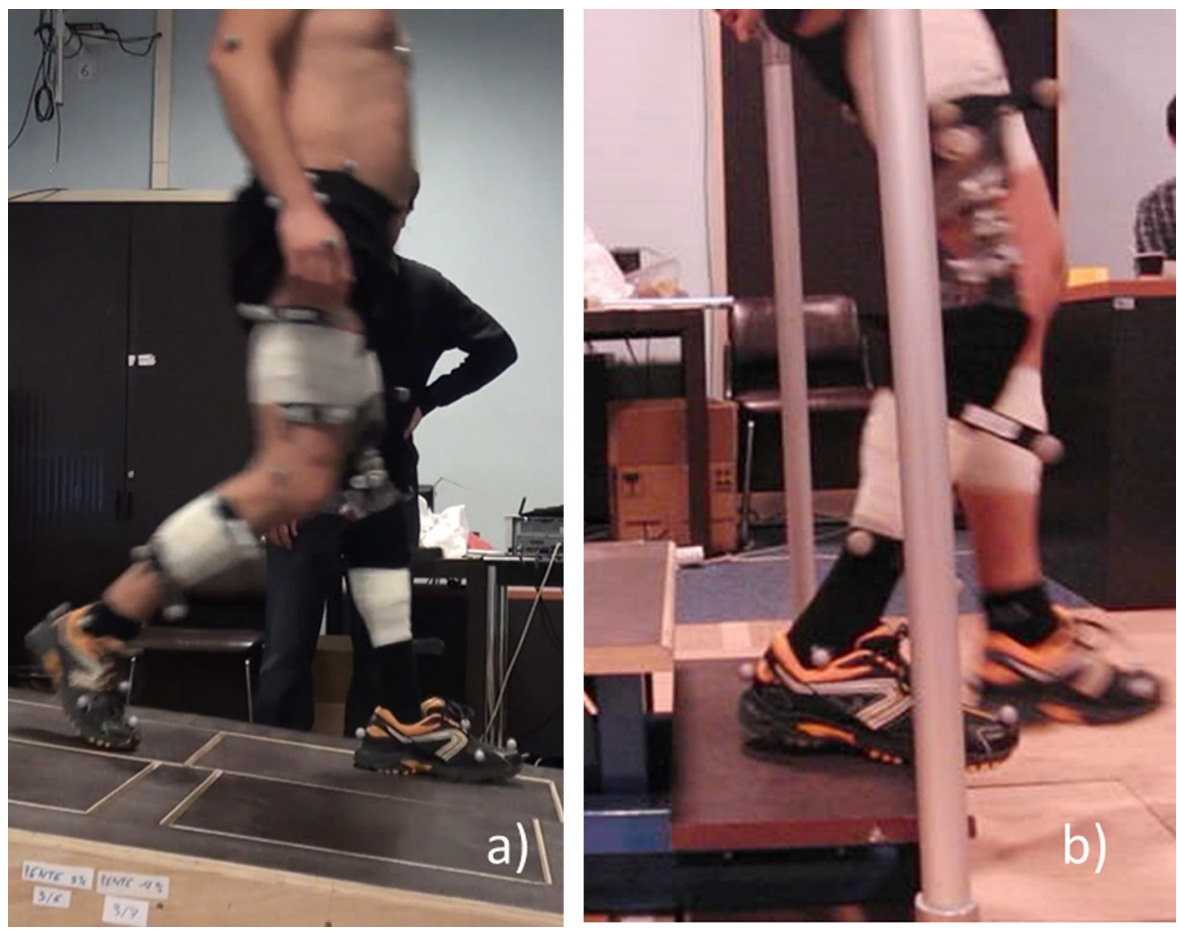

Fig. 4. Patient fitted with the prototype during (a) slope descent and (b) ramp descent.

feet does not allow step-by-step descent of a stair in the same way as non-amputee people do. Thus, the progression in the stair is only possible by placing the prosthetic foot on the edge of the step inducing high falling risk. Proposing innovative technological solutions should address this issue in order to fit patients 'expectations.

During the project, a prototype of an ankle-knee prosthetic system was designed using this approach [16]. Briefly, the ankle and the knee were controlled by two hydraulic cylinders driven by a microprocessor through motorized valves, which can adapt the resistance or the range of motion of the joints. Embedded sensors (inertial, joint, force) information were used by the microprocessor to localize the prosthetic limb, detect the different activities (standing, walking, slopes, stairs, etc.) and adapted the behavior of the prosthetic limb. (Fig. 4)

\section{Conclusion and perspectives}

The project APSIC, through extensive motion analysis study, succeeded in gaining knowledge about functional contributions of joints during the locomotion in situations of daily living and about the impairments and compensations of people with amputation. The innovative prosthetic ankle-knee is being evaluated by comparison with existing devices. Data were also used to provide new approach of rehabilitation by quantifying gait defaults such as vaulting for example and by defining personalized guidelines adapted to each walking situation.

\section{Disclosure of interest}

X.B., F.D., J.M. and P.G. are employees of Proteor.

\section{Acknowledgement}

This study was supported by the French National Research Agency, under reference ANR- 2010-TECS-020.

\section{References}

[1] Samuelsson KAM, Töytäri O, Salminen AL, Brandt A. Effects of lower limb prosthesis on activity, participation, and quality of life: a systematic review. Prosthet Orthot Int 2012;36(2):145-58

[2] Sawers AB, Hafner BJ. Outcomes associated with the use of microprocessor-controlled prosthetic knees among individuals with unilateral transfemoral limb loss: a systematic review. J Rehabil Res Dev 2013;50(3):273-314.

[3] Lay AN, Hass CJ, Gregor RJ. The effects of sloped surfaces on locomotion: a kinematic and kinetic analysis. J Biomech 2006;39(9):1621-8.

[4] Silder A, Besier T, Delp SL. Predicting the metabolic cost of incline walking from muscle activity and walking mechanics. J Biomech 2012;45(10):1842-9.

[5] Riener R, Rabuffetti M, Frigo C. Stair ascent and descent at different inclinations. Gait Posture 2002;15(1):32-44.

[6] Dixon PC, Pearsall DJ. Gait dynamics on a cross-slope walking surface. J Appl Biomech 2010;26(1):17-25.

[7] Della Croce U, Bonato P. A novel design for an instrumented stairway. J Biomech 2007;40(3):702-4.

[8] Langlois K, Villa C, Bonnet X, Lavaste F, Fodé P, Martinet $\mathrm{N}$, et al. Influence of transtibial amputee physical capacities on gait adjustments on sloped surface. J Rehabil Res Dev 2014, http://dx.doi.org/10.1682/JRRD.2013.05.0118.

[9] Dumas R, Chèze L, Verriest JP. Adjustments to McConville et al. and Young et al. body segment inertial parameters. J Biomech 2007;40(3):543-53.

[10] Wu G, Siegler S, Allard P, Kirtley C, Leardini A, Rosenbaum D, et al. ISB recommendation on definitions of joint coordinate system of various joints for the reporting of human joint motion - part I: ankle, hip, and spine. International Society of Biomechanics. J Biomech 2002;35(4):543-8. 
[11] Pillet H, Bonnet X, Lavaste F, Skalli W. Evaluation of force plate-less estimation of the trajectory of the centre of pressure during gait. Comparison of two anthropometric models. Gait Posture 2010;31(2):147-52.

[12] Wu G, Cavanagh PR. ISB recommendations for standardization in the reporting of kinematic data. J Biomech 1995;28(10):1257-61.

[13] Robertson DG, Winter DA. Mechanical energy generation, absorption and transfer amongst segments during walking. J Biomech 1980;13(10):845-54.
[14] Hansen AH, Childress DS, Knox EH. Roll-over shapes of human locomotor systems: effects of walking speed. Clin Biomech (Bristol, Avon) 2004;19(4):407-14.

[15] Pitkin M. What can normal gait biomechanics teach a designer of lower limb prostheses? Acta Bioeng Biomech 2013;15(1):3-10.

[16] Bonnet X, Djian F. Hydraulic system for a knee-ankle assembly controlled by a microprocessor. $2014 \#$ \#jan\# 30. WO Patent App. PCT/EP2013/065,845. 